\title{
Micromagnetic simulations of magnetoelectric materials
}

\author{
Thomas Fischbacher, Matteo Franchin, Hans Fangohr ${ }^{1}$ \\ ${ }^{1}$ School of Engineering Sciences, University of Southampton, SO17 1BJ Southampton, United Kingdom
}

Some magnetic materials show a magnetoelectric coupling between inhomogeneous magnetization patterns and electric polarization that is sufficiently strong to allow external control of magnetization structure by electric fields. Numerical simulations of 'magnetoelectric' materials of this type require an extension of the standard micromagnetic model which conceptually parallels the introduction of spin-current interaction terms. We show how the micromagnetic simulator 'Nmag' can be extended to support the inhomogeneous magnetoelectric interaction term and also give a simple self-contained example for simulating the micromagnetic dynamics of a magnetoelectric system in the presence of an external electric field.

\section{INTRODUCTION}

Magnetoelectric behaviour is related to the existence of a coupling term in the Free Energy functional that links the material's electric and magnetic polarizabilities. Generically, such terms are expected to arise due to spin-orbit coupling - unless forbidden by some (here: crystal) symmetry. As the ability to easily control magnetization structures by electric fields (and vice versa) would be very appealing from an application perspective, a key question is which materials exhibit a sufficiently strong magnetoelectric effect to make this feasible.

Generally, magnetoelectric behaviour can in principle arise not only in ferroelectric-ferromagnetic materials (multiferroics) where it is most often observed, but also e.g. in paramagnetic ferroelectrics or non-ferroelectric ferromagnets (cf. [1-3] for history and terminology). The first demonstration of the time reversal symmetry violating homogeneous magnetoelectric effect was given by Dzyaloshinskii in 1960 [4], while electric polarization of Neel domain walls - the inhomogeneous effect relevant for this work - was predicted in [5]. A magnetoelectric effect of considerable strength can in particular arise in composite materials, where it may be mechanically mediated through piezoelectric-magnetostrictive coupling. While the simulation as well as the production of magnetoelectric composites for device applications poses a number of interesting research challenges, it recently has been demonstrated that even in some single-phase magnetoelectric materials (such as some suitably grown iron garnets), electric control of micromagnetic magnetization structure via the magnetoelectric effect is feasible at room temperature [6]. Considering a systematic approach towards micromagnetic simulations of magnetoelectric materials, it certainly is attractive to start from the simplest possible model that is of wider scientific interest and then gradually extend this by taking other effects into account - in continuation of the spirit of the original micromagnetic model. Such a philosophy suggests to first tackle the simulation of magnetoelectric materials whose behaviour can be described without having to introduce extra dynamical degrees of freedom beyond the magnetic ones, before this then is extended to ferroelectric materials (with additional polarization degrees of freedom) and beyond.

Naturally, any modeling approach of some particular effect that is based on taking the corresponding leading order correction term(s) in a Ginzburg-Landau ansatz for the Free Energy into account will make choices about terms that can be dropped. These choices usually depend on simplifying properties of the specific system under study. This makes the idea of a generic 'magnetism $+\mathrm{X}$ ' simulation framework appear very attractive which handles extensions to the micromagnetic model by being reconfigurable at the level of field theoretic equations. As the free micromagnetic simulation package Nmag (that has been developed by the authors, [7]) supports this concept by design, we will in the following describe how the simulation of a magnetoelectric material for the simplest possible case that is physically interesting can be done with Nmag.

\section{THE MODEL}

Simple considerations about the behaviour of thermodynamic potentials under time and space reflections show that the leading order magnetoelectric terms must be quadratic in the magnetization $\vec{M}$ and can be linear in the polarization $\vec{P}$ if accompanied by an additional spatial derivative. In the simplest case, the leading magnetoelectric contribution will be of the form (cf. e.g. [8]):

$$
F_{M E}=\int d^{3} x \gamma P_{j}\left(M_{j} \partial_{k} M_{k}-M_{k} \partial_{k} M_{j}\right)
$$

In order to simplify this even further, we take the polarization $\vec{P}$ to be proportional to the externally applied electric field. The extension to non-linear relations between the externally applied electric field $\vec{E}_{\text {ext }}$ and polarization $\vec{P}$ will not be discussed in detail here - in the simplest case, we can consider $\vec{P}$ to be given (rather than $\vec{E})$.

We then arrive at:

$$
F_{M E}=\int d^{3} x \tilde{\gamma} E_{j}\left(M_{j} \partial_{k} M_{k}-M_{k} \partial_{k} M_{j}\right)
$$


with some externally specified field $\vec{E}$ (or instead, $\vec{P}$ ).

Taking variational derivatives of this expression at specific locations $\delta / \delta M_{j}\left(x_{k}\right)$ gives an extra contribution $\vec{H}_{M E}$ to the total effective magnetic field $\vec{H}_{\text {total }}$ which conceptually resembles the exchange field $\vec{H}_{\text {exch }}$. This field strength is:

$$
H_{\mathrm{ME}, i}=2 \tilde{\gamma} M_{\mathrm{sat}}\left(E_{i} \mu_{j j}-E_{j} \mu_{j i}\right)
$$

where the rank-2 tensor $\mu_{i j}$ is an auxiliary intermediate quantity defined as:

$$
\mu_{i j}:=\partial_{j} m_{i} \quad\left(m_{i}=M_{i} / M_{\mathrm{sat}}\right)
$$

Evidently, in addition to the introduction of this new field $\vec{H}_{\mathrm{ME}}$, the micromagnetic simulator also must be extended to support the applied electric field $\vec{E}_{\text {ext }}$ as well as the vector gradient of the magnetization. In Nmag, this is comparatively straightforward, in particular as the package internally supports tensor fields of arbitrary rank. Incidentally, the calculation of $\mu_{i j}$ already has been implemented before for Nmag's spin torque capabilities.

\section{SIMULATION AND RESULTS}

The specific toy system to be simulated is a $200 \mathrm{~nm} \times 100 \mathrm{~nm} \times 10 \mathrm{~nm}$ slab of some fictitious magnetoelectric material whose properties have been chosen in order to permit an example that neither requires much memory nor CPU time (well below 1.5 GB and 5 hours on a recent system using one $\mathrm{CPU}$ ). The edges of this block lie parallel to the coordinate axes, and two opposite corners are given by (all coordinates in $\mathrm{nm})(-100,0,0)$ and $(100,100,10)$. In addition, there is a long electrically charged wire at $(0,-500,0)$ running in $(0,0,1)$-direction, which produces an (inhomogeneous) electric field that falls off with distance from the wire as $1 /|\vec{r}|$. In this example, the maximum field strength occurs at $(0,0,0)$, with a value of $\pm 2 \cdot 10^{6} \mathrm{~V} / \mathrm{m}$ in $+y$-direction (well below the dielectric breakdown voltage of most materials). Along the faces that are perpendicular to the $y$-axis, the slab's magnetization is 'pinned' to point in $\pm x$-direction (for $y=0$ and $y=100$ respectively). The calculation starts by determining the equilibrium domain wall configuration under these conditions from an initial magnetization configuration that gradually rotates in the $x-y$ plane (angle proportional to $y$-coordinate), with a small $+z$-component to break the artificial alignment with the coordinate axes. Then, an electric field is turned on that has a maximal value of $+2 \mathrm{MV} / \mathrm{m}$ in $+y$-direction at $(0,0,0)$ and the new equilibrium configuration is calculated. Afterwards, the electric field is reversed and the magnetization configuration is again allowed to relax. Starting from this particular initial magnetization configuration, the film relaxes into a Y-like branched domain wall structure of (locally) minimal energy. The "tail" of the Y-configuration then reacts fairly strongly to an externally applied electric field.

Due to the structure of the $E M \partial M$ term, neither spatially homogeneous magnetizations nor Bloch walls couple to electric fields, while Neel wall type configurations do. In thin films, shape anisotropy will hence play an important role for the occurrence of this effect, but more generically, magnetocrystalline anisotropy may also be employed to achieve this, as in [6]. For this reason, a weak uniaxial anisotropy that is not aligned with the axes of the simulated sample but has its easy axis pointing in the $(0,1,1)$ direction has been included in the example. The material parameters are: $\left(M\right.$ sat, $\left.J, K_{1}, \tilde{\gamma}\right)=$ $\left(0.5 \mathrm{MA} / \mathrm{m}, 10^{-12} \mathrm{~J} / \mathrm{m}, 100 \mathrm{~J} / \mathrm{m}^{3}, 3 \cdot 10^{9} 1 / \mathrm{As}\right)$. In order to speed up the relaxation process, the LLG precession term has been turned off.

As the magnetoelectric extension to Nmag is in an early development stage, the code for this extension has been separated off into an experimental/evolving branch for which no user support guarantees can be given. To the Nmag user, this means that in order to use the magnetoelectric extension, the simulation script can use standard Nmag but should not start with the line 'import nmag', but instead:

\section{import nmag.nmag_magnetoelectric as nmag}

The complete script is shown in figure 1. After defining the material (lines 05-16), it sets up a simulation (line 18), loads the mesh (lines 19-20), and uses three auxiliary functions fun_pin, fun_m, fun_E (lines 24-42) to set the (inhomogeneous) 'pinning field', initial magnetization, and electric field (lines 44, 45, 47, 49). Special attention has to be paid to fun_E: as this takes an extra field strength argument, but sim.set_Electric_ext() has to provide a function of the coordinates only, we use an anonymous lambda function to fix the first (field strength) parameter (lines 47 and 49).

The simulation results are shown in figure 2: Depending on polarity, the wire can either attract or repel the domain wall, in qualitative accordance with [6]. Simulations of more realistic problems typically will involve wider domain walls and hence require larger meshes.

\section{CONCLUSIONS AND OUTLOOK}

The toy model presented here exemplifies how to use an experimental extension to the Nmag micromagnetic simulator (that gets shipped with the standard codebase) to set up micromagnetic calculations with magnetoelectric coupling for more involved systems than the one presented here. While the toy example utilizes non-physical parameter choices to minimize the size of the problem, it qualitatively reproduces behaviour that has been observed experimentally in [6]. As usual with Nmag, this 
01 import nmag.nmag_magnetoelectric as nmag

02 from nmag import SI

03 import math

04

$05 \mathrm{ME}=\mathrm{nmag}$. MagMaterial (name $=$ "ME",

$06 \quad$ Ms=SI $(5 e 5, " \mathrm{~A} / \mathrm{m} ")$,

07 exchange_coupling $=\backslash$

$08 \quad \mathrm{SI}(1 \mathrm{e}-12, \mathrm{~J} / \mathrm{m} ")$

09 anisotropy=

10 nmag.uniaxial_anisotropy (

axis $=[0,1,1], \backslash$

$\left.\mathrm{K} 1=\mathrm{SI}\left(100, \mathrm{~J} / \mathrm{m}^{\wedge} 3 "\right)\right)$

magnetoelectric_coupling $=\backslash$ $\mathrm{SI}(3 \mathrm{e} 9, " 1 / \mathrm{A}$ s"),

llg_damping $=0.5$,

do_precession=False)

8 sim=nmag.Simulation ("ME_example")

sim.load_mesh("ME_mesh.nmesh.h5", [("ME",ME)]

20 unit_length=SI (1e-9, "m"))

21 \# This is a slab with these dimensions (in $\mathrm{nm}$ ):

$2(\operatorname{dim} X, \operatorname{dim} Y, \operatorname{dimZ})=(200,100,10)$

4 \# Cf. Manual example: Pinning Magnetisation

25 def fun_pin(coords):

if coords $[1]<1 * 1 \mathrm{e}-9\rangle$

or coords $[1]>(\operatorname{dim} Y-1) * 1 e-9$ :

return 0.0

return 1.0

def fun_m(coords):

$\mathrm{h}=\operatorname{coords}[1] / 1 \mathrm{e}-9$

return [math.cos (math.pi*h/dimY),

math.sin (math.pi*h/dimY),

1e-6] \# to break the symmetry

def fun_E(EO, coords):

$\left(x_{-} n m, y_{-} n m\right)=(\operatorname{coords}[0] / 1 e-9, \operatorname{coords}[1] / 1 e-9+500)$

$\mathrm{r}_{-} \mathrm{nm}=\left(\mathrm{x} \_\mathrm{nm} * * 2+\mathrm{y} \_\mathrm{nm} * * 2\right) * * 0.5$

return $\left[\mathrm{E} 0 * \mathrm{x}_{-} \mathrm{nm} / \mathrm{r} \_\mathrm{nm} * * 2\right.$,

$\mathrm{E} 0 * \mathrm{y} \_\mathrm{nm} / \mathrm{r}_{-} \mathrm{nm} * * 2$,

$0.0]$

43

44 sim.set_pinning (fun_pin)

45 sim.set_m(fun_m)

46 sim.relax()

47 sim.set_Electric_ext (lambda c:fun_E $(+1 e 9, c), S I(1, " V / m "))$

48 sim.relax ()

49 sim.set_Electric_ext (lambda c:fun_E $(-1 \mathrm{e} 9, \mathrm{c}), \mathrm{SI}(1, \mathrm{~V} / \mathrm{m} ")$ )

50 sim.relax()

FIG. 1: The Nmag simulation script

extension feature is fully compatible with MPI-parallel execution, as described in [9].

Considering future extensions of this basic model which then will also have to take electrical polarization dynamics into account, the task of extending the basic micromagnetic model in Nmag would greatly benefit from an extension to the Nsim [7] simulation core (on top of which Nmag is implemented) that automatically generates the field dependency tree from the user-supplied physical field equations. This tree is used internally to find out which dependent fields have become invalid after a field update and need to be re-computed (automatically) before they can be read. So far, this has to be specified manually for every new extension.

Acknowledgments This work was supported in part by EPSRC grant EP/E040063/1.
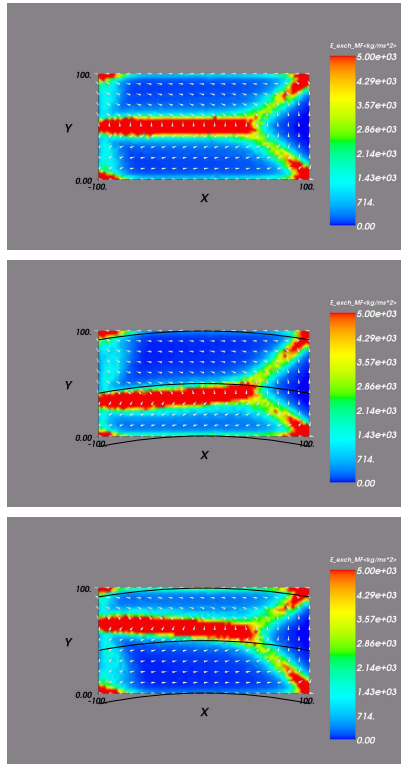

FIG. 2: Exchange energy density (color coded, color online) and magnetization structure for $E_{\max , y}=0,+2,-2 \mathrm{MV} / \mathrm{m}$ (top to bottom). For the electric field strengths and material parameters used in this example, the open end of the domain wall on the left $\mathrm{y}-\mathrm{z}$ face moves by about $17 \mathrm{~nm}$ in either direction. Electric equipotential surfaces are shown as black arcs, the (perpendicular) field strengths being $\pm 1.67, \pm 1.82$ and $\pm 2.00 \mathrm{MV} / \mathrm{m}$ (outer to inner).

[1] W. Eerenstein, N. D. Mathur, J. F. Scott: 'Multiferroic and magnetoelectric materials', Nature 442, 759-765 (17 August 2006), doi:10.1038/nature05023

[2] D. Khomskii, 'Classifying multiferroics: Mechanisms and effects, Physics 2, 20 (2009) doi:10.1103/Physics.2.20

[3] M. Fiebig, 'Revival of the magnetoelectric effect, J. Phys. D: Appl. Phys. 38 (2005) R123R152 doi: $10.1088 / 0022-3727 / 38 / 8 /$ R01

[4] I. E. Dzyaloshinskii, 'On the magneto-electrical effect in antiferromagnets', Sov. Phys. JETP 10, 628 (1960)

[5] V.G. Bar'yakthar, V.A. L'vov, D.A. Yablonskiř, 'Inhomogeneous magnetoelectric effect', JETP Lett 37, 673 (1983)

[6] A. S. Logginov, G. A. Meshkov, A. V. Nikolaev, E. P. Nikolaeva, A. P. Pyatakov, A. K. Zvezdin, 'Room temperature magnetoelectric control of micromagnetic structure in iron garnet films' Apl. Phys. Lett. 93, 182510 (2008) doi:10.1063/1.3013569

[7] T. Fischbacher, H. Fangohr, 'Continuum multi-physics modeling with scripting languages: the Nsim simulation compiler prototype for classical field theory', arXiv: 0907.1587

[8] M. Mostovoy, 'Ferroelectricity in Spiral Magnets', Phys. Rev. Lett. 97, 067601 (2006) doi:10.1103/PhysRevLett.96.067601

[9] T. Fischbacher, M. Franchin, G. Bordignon, A. Knittel, H. Fangohr, 'Parallel execution and scriptability in micromagnetic simulations', J. Appl. Phys. Vol. 105 Issue 7, 07D527 - 07D527-3 (2009) doi:10.1063/1.3073937 